\title{
NOTES
}

\section{Degradation of Aliphatic Polyesters by Vacuum Ultraviolet Irradiation}

\author{
Yabin Li, Akiko HaYASHI, Mizuho SAITO, Martin VACHA, \\ Shigemitsu MURASE, and Hisaya SATO ${ }^{\dagger}$ \\ Graduate School of Bio-Application Systems and Engineering, Tokyo University \\ of Agriculture \& Technology, 2-4-16 Naka-cho, Koganei 184-8588, Japan
}

(Received September 27, 2005; Accepted November 17, 2005; Published April 15, 2006)

\author{
KEY WORDS Aliphatic Polyesters / Vacuum Ultraviolet Irradiation / Degradation / Negatively \\ Charged Oxygen / \\ [DOI 10.1295/polymj.38.395]
}

The photon energy of vacuum ultraviolet (VUV) light is higher than the binding energy of some chemical bonds in organic molecules, such as $\mathrm{C}-\mathrm{C}, \mathrm{C}-\mathrm{H}$, or $\mathrm{C}-\mathrm{O}$. Therefore, VUV light is able to cause the scission of various bonds in polymer molecules, which can lead to the decomposition of the polymeric molecules. Degradation of polymers by VUV has been studied with the purpose of investigating damage to polymeric materials by VUV, developing new photoresist materials, revealing the degradation mechanism, and modifying polymer surfaces, etc. ${ }^{1-9}$ The degradation mechanism of poly(methyl methacrylate) (PMMA) has been extensively studied for evaluation as a new photoresist material. ${ }^{10-13}$ It was found that the side chain ester group is decomposed by VUV irradiation, leading to a polymer containing carboncarbon double bonds. In addition, scission of the main chain also occurred. If an ester group is easily cleaved by VUV, it may be expected that aliphatic polyesters, which contain ester groups in the main chain, will be degraded to small volatile molecules and may function as good photoresist materials. Aliphatic polyesters are soluble in common organic solvents, which enables good thin films to be easily formed. It is well known that aliphatic polyesters are easily degraded by biological reactions, and although many studies have been performed regarding the biodegradation of aliphatic polyesters, only a few studies have investigated degradation by irradiation with UV or VUV light. In this report, the degradation of aliphatic polyesters by VUV was investigated and compared with that of PMMA. The study concentrated on the reaction mechanism, the effect of oxygen and application of an external electric field during irradiation.

\section{EXPERIMENTAL}

\section{Materials}

Poly(methyl methacrylate) (PMMA), poly(L-lactic acid) (PLA), and poly(butylene succinate) (PBS) were obtained commercially and used without further purification.

\section{Methods}

Polymer thin films were prepared by spin coating a chloroform solution of the respective polymer $(0.010$ $\mathrm{g} \mathrm{mL}^{-1}$ ) on a germanium or quartz glass substrate at a spin rate of $500 \mathrm{rpm}$. The film thickness was in the order of $0.2 \mu \mathrm{m}$. For the patterning experiment the film was heated at $80^{\circ} \mathrm{C}$ for $10 \mathrm{~min}$.

VUV was generated using a Xe excimer lamp (Harrison Toshiba Lighting) with a center wavelength of $172 \mathrm{~nm}$. The light intensity was $0.23 \mathrm{~mW} \mathrm{~cm}^{-2}$ as measured at the sample position with a power meter (Ushio, UIT-150) and a detector (Ushio, UVD-C254). IR spectra were measured for the samples on germanium substrates using a Jasco FT-IR-460 spectrometer. UV spectra were measured for the samples on quartz glass substrates using a Jasco V-570 spectrometer. Surface profiles were observed using a contact profilometer (Dektak). Micrographs were obtained using a digital microscope (Keyence, VH-Z450).

\section{RESULTS AND DISCUSSION}

The degradation of the polymers upon irradiation with the Xe excimer lamp was characterized by IR spectroscopy. Figure 1 shows an example of the IR spectral change of PBS film with the thickness of $0.4 \mu \mathrm{m}$. The intensities of all the peaks decreased uni-

${ }^{\dagger}$ To whom correspondence should be addressed (Tel: +81-42-388-7050, Fax: +81-42-388-7050, E-mail: h-sato@cc.tuat.ac.jp). 


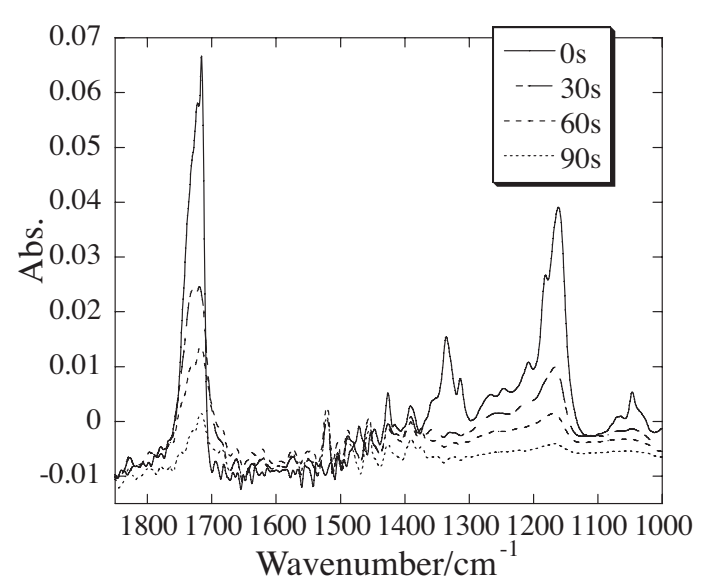

Figure 1. Change of FT-IR spectra for PBS polymer film under VUV irradiation.

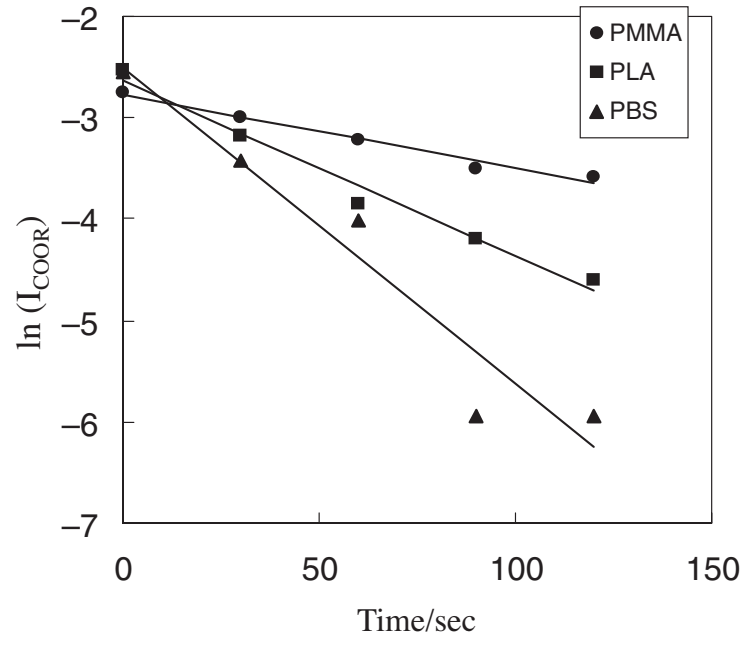

Figure 2. Decrease of carbonyl absorption of polymers. A and A0 indicate the carbonyl absorptions for irradiated and non-irradiated polymers, respectively.
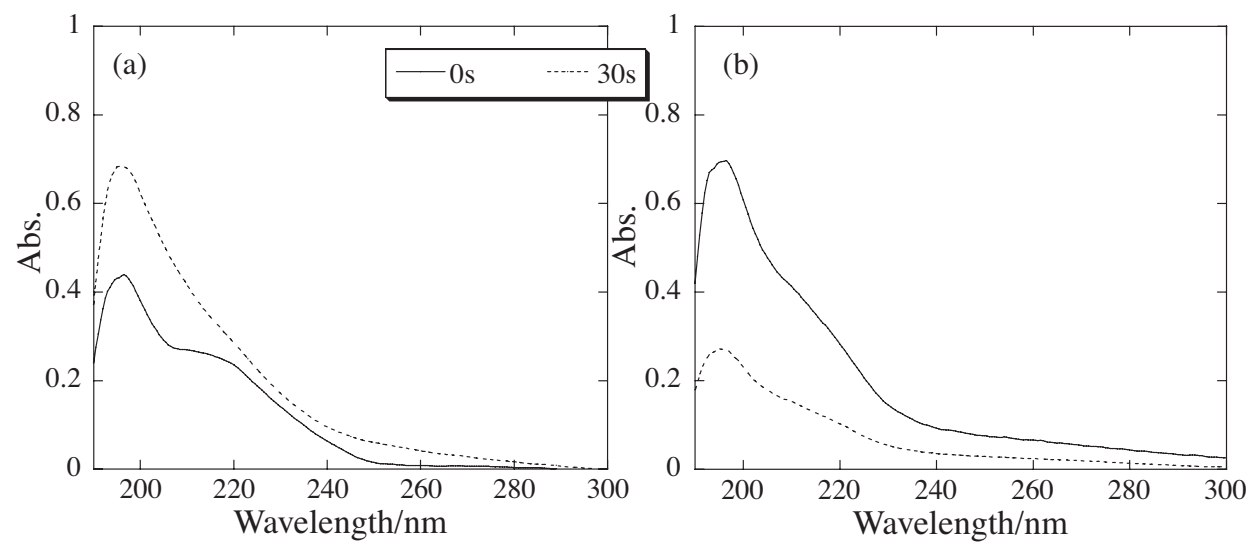

Figure 3. Change of UV spectra for (a) PMMA and (b) PBS under VUV irradiation for $30 \mathrm{~s}$.

formly with irradiation, indicating that the polymers were mainly decomposed to small volatile molecules. The ratio of the carbonyl stretching signal intensity for the irradiated to non-irradiated sample was calculated in the range of 1720 to $1750 \mathrm{~cm}^{-1}$. Figure 2 shows the $\log$ of the ratio plotted against irradiation time, and the results gave straight lines for all the polymers examined. The negative values of the slopes correspond to the degradation rates, and were $7.2 \times 10^{-3}, 17 \times$ $10^{-3}$, and $31 \times 10^{-3} \mathrm{~s}^{-1}$, for PMMA, PLA, and PBS, respectively. Therefore, it was confirmed that both aliphatic polyesters studied have degradation rates higher than PMMA.

Figure 3 compares the UV spectral changes of PMMA and PBS. In the case of PMMA, irradiation with VUV caused an increase of the main absorption band at approximately $200 \mathrm{~nm}$, and at the same time a new absorption shoulder appeared superimposed at the longer wavelength end of the peak at $220 \mathrm{~nm}$. This absorption increase can be attributed to the production of conjugated double bonds in the main chain due to the scission of side chains. Such production of conjugated double bonds has also been reported by other groups. $^{7-9}$ On the other hand, PBS showed an overall decrease in UV absorption. This observation indicates that for PBS, the scission of ester groups occurs on the main chain and leads to the production of small volatile molecules.

In the patterning experiments, the polymer film with $0.2 \mu \mathrm{m}$ was irradiated with VUV through a mask consisting of five strips with widths of 1000, 500, 250, 125 , and $62.5 \mu \mathrm{m}$, and with a $500 \mu \mathrm{m}$ spacing between the strips. If the mask was attached to the film surface, the degradation was very slow. Therefore, a $10 \mu \mathrm{m}$ spacer was inserted between the mask and the film. Figure 4 shows the surface pattern after irradiation for $30 \mathrm{~s}$. PBS showed almost complete etching and the irradiated part revealed the flat surface of the glass. However, the non-irradiated part was also slightly etched, leaving an uneven surface. Irradiation 


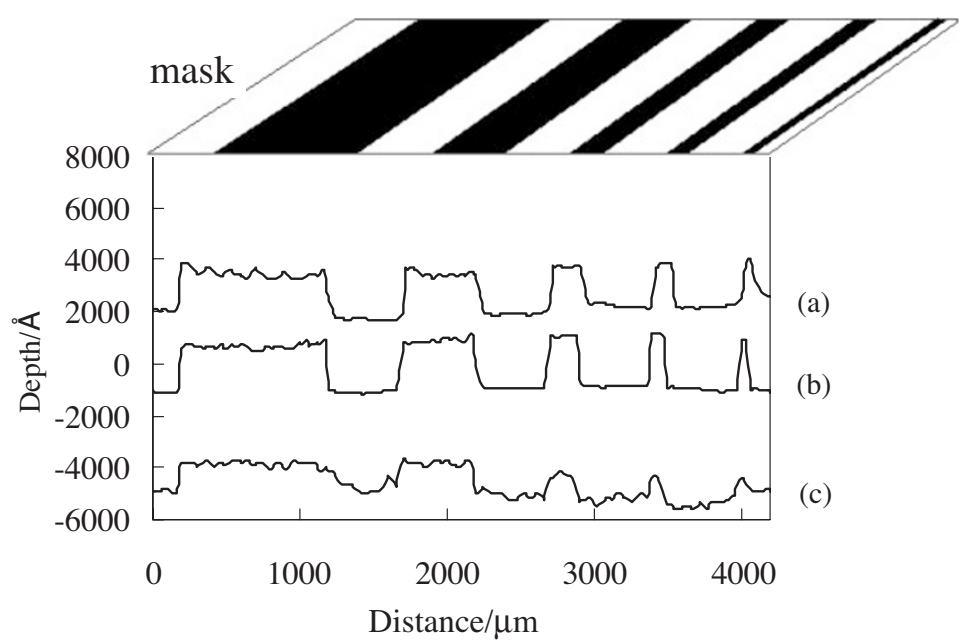

Figure 4. Surface profile of polymers after irradiation with VUV through a photomask for 30 s. (a) PLA, (b) PBS and (c) PMMA.

Table I. Degradation rate of PBS under different atmosphere

\begin{tabular}{lrllcc}
\hline Atmosphere & Air & $\begin{array}{c}\text { Air: } \\
\text { 3 torr }\end{array}$ & Nitrogen & \multicolumn{2}{c}{ Air: under electric field } \\
\cline { 5 - 6 } & & & Top $(-)$ & Top $(+)$ \\
\hline Rate $/ 10^{-3} \mathrm{~s}^{-1}$ & 31 & 7.6 & 3.5 & 53 & 5.7 \\
Relative rate & 1 & 0.25 & 0.11 & 1.7 & 0.18 \\
\hline
\end{tabular}

of masked PLA gave almost the same results as PBS. On the other hand, PMMA did not show a clear etching pattern. This finding can be explained by a slower decomposition rate, and also by the fact that a portion of the main chains of the irradiated part were either not degraded or produced non-volatile materials. This can be explained by the preferential scission of the side chains, which leaves the double bond on the main chain intact.

From the results of Figure 4, we assumed that surface roughening of the non-irradiated parts might be caused by activated species that are generated at the VUV irradiated part and migrate to the non-irradiated part. In order to determine the type of active species involved, the degradation rate of PBS was measured in vacuum (more specifically, under a reduced pressure of 3 torr), in air, and in a nitrogen atmosphere. Table I shows the effect of the atmosphere on the degradation rate of PBS. The degradation rates were found to be $3.5 \times 10^{-3}, 7.6 \times 10^{-3}$, and $31 \times 10^{-3}$ $\mathrm{s}^{-1}$, for vacuum, nitrogen, and atmospheric air conditions, respectively. The decrease in the reaction rate to almost one tenth and one fifth, when in vacuum and under nitrogen atmosphere, respectively, indicates that irradiated oxygen is the activated species responsible for the degradation. This observation agrees with the results of several other groups who also found that oxygen or atmospheric pressure increases the rate of photoreaction. $8,13,14$

If the activated species are ionic, then migration could be controlled by an external electric field. To
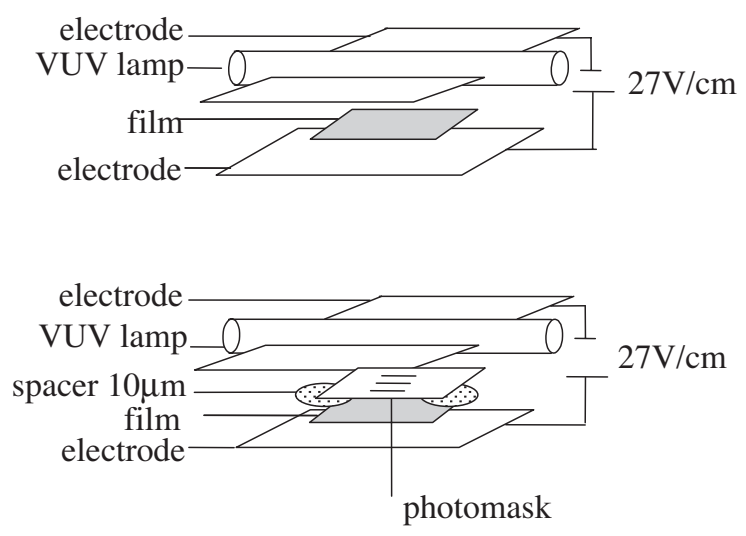

Figure 5. Set-up image under an electric field with photomask (a) and without photomask (b). The distance between the two electrodes is $10 \mathrm{~mm}$, and the distance between the lamp and film surface is $2 \mathrm{~mm}$. The thickness of the photomask is $1 \mathrm{~mm}$. The diameter of the lamp is $20 \mathrm{~mm}$.

examine this assumption, degradation by VUV light was performed on a PBS sample under an electric field of $27 \mathrm{~V} \mathrm{~cm}^{-1}$, as shown schematically in Figure 5. The degradation rates with and without an applied field were measured in air and are compared with the results obtained in a nitrogen atmosphere (Table I). When the electric field is oriented from top $(+$ on the upper electrode) to bottom ( - on the lower electrode), the degradation rate decreases to $5.7 \times 10^{-3}$ $\mathrm{s}^{-1}$, which is almost the same rate as that obtained under a nitrogen atmosphere without an electric field. This finding indicates that positive or neutral species were much less influential in the degradation process. On the other hand, the degradation rate increased to $53 \times 10^{-3} \mathrm{~s}^{-1}$ when the direction of the electric field was reversed. Therefore, it is very likely that negatively charged oxygen species, produced by VUV irradiation, are the activated species responsible for the degradation. 


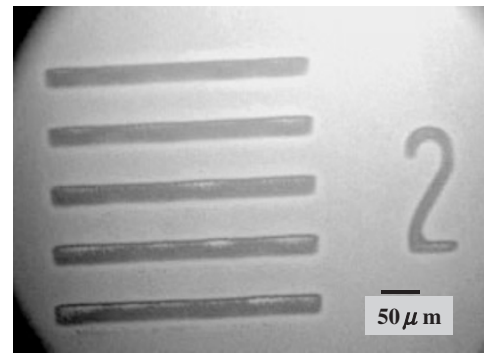

Surface image

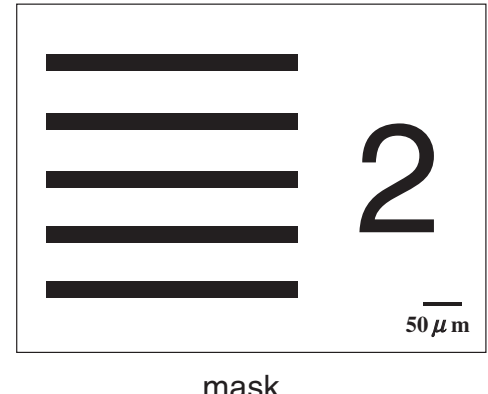

mask

Figure 7. Image of PBS surface after VUV irradiation for $30 \mathrm{~s}$ under an electric field from top (+) to bottom (-).

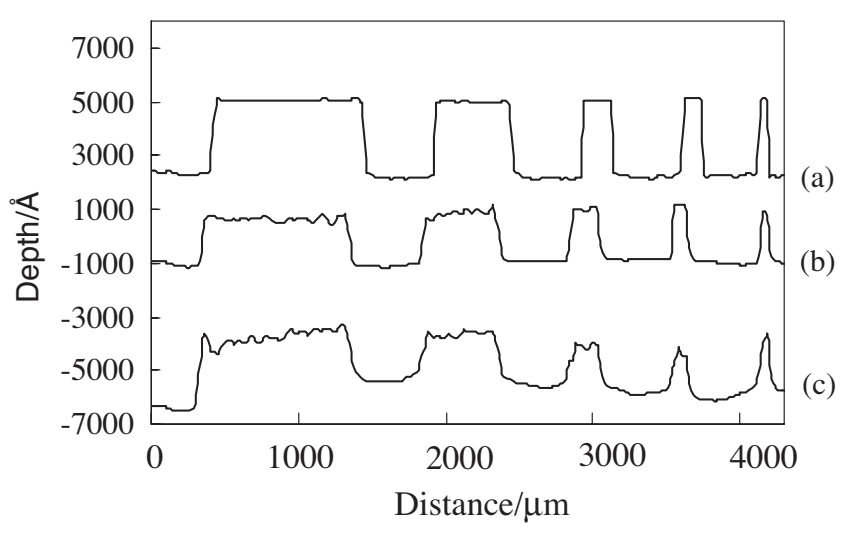

Figure 6. Surface profile of PBS after VUV irradiation for $30 \mathrm{~s}$ under an electric field. (a) Electric field from top (+) to bottom (-), (b) no electric field, and (c) electric field from bottom $(+)$ to top (-).

The patterning experiment was repeated on the PBS sample under an electric field of $27 \mathrm{~V} \mathrm{~cm}^{-1}$. As shown in Figure 5, when the electric field was directed from bottom to top, the masked area showed a clean flat surface with sharp vertical edges, and the irradiated area was etched down to the glass surface. This result can be understood by assuming that the negatively charged activated oxygen, which is generated in the irradiated areas, is transferred directly downward without migrating horizontally to the masked regions. This downward transfer causes acceleration of the degradation at the etching area and prevents roughening of the masked regions. On the other hand, with the opposite bias, the pattern shows varying height and conical edges as a result of diffusion of the activated species. Furthermore, a considerable amount of polymer remained at the etched area, indicating slower degradation.

As the results in Figure 6 show a clearly etched profile, even for the narrowest strip $(62.5 \mu \mathrm{m})$ the electric field assisted patterning experiment was also carried out using a mask with strips of $40 \mu \mathrm{m}$ in width and with the electric field directed from bottom to top. As shown in Figure 7, a clearer pattern with good contrast was obtained. The roughness of the edge was approximately 1 to $2 \mu \mathrm{m}$. If a parallel light source is used, then better resolution may be obtained. The results show that aliphatic polyesters are able to provide a good photoresist material when using VUV irradiation.

\section{CONCLUSIONS}

Aliphatic polyesters such as PBS and PLA revealed a higher degradation rate than PMMA under VUV irradiation. It was found that the active species for degradation by VUV irradiation was negatively charged oxygen, and the degradation by VUV light alone is only $10 \%$ of that due to the activated species. With an electric field applied from the bottom (film side) to the top (lamp side), a good patterning was obtained for a $0.2 \mu \mathrm{m}$ thick film.

Acknowledgment. The authors are grateful to Harison Toshiba Lighting Co. for financial support.

\section{REFERENCES}

1. R. Wilken, A. Hollaender, and J. Behnisch, Plasmas Polym., 7, 185 (2002).

2. A. C. Fozza, J. E. Klemberg-Sapieha, and M. R. Wertheimer, Plasmas Polym., 4, 183 (1999).

3. G. Peng, W. Hao, D. Yang, and S. He, J. Appl. Polym. Sci., 94, 1370 (2004).

4. V. E. Skurat, P. V. Samsonov, and A. P. Nikiforov, High Perform. Polym., 16, 355 (2004).

5. J. A. Dever and C. A. McCracken, High Perform. Polym., 16, 289 (2004).

6. a) A. Hozumi, N. Shirahata, Y. Nakanishi, S. Asakura, and A. Fuwa, J. Vac. Sci. Technol., A, 22, 1309 (2004).

b) N. A. Bullett, D. P. Bullett, F.-E. Truica-Marasescu, S. Lerouge, F. Mwale, and M. R. Wertheimer, Appl. Surf. Sci., 235, 395 (2004).

7. F.-E. Truica-Marasescu and M. R. Wertheimer, Macromol. Chem. Phys., 206, 744 (2005).

8. M. Tagawa, K. Yokota, and N. Ohmae, J. Spacecraft and Rockets, 41, 345 (2004).

9. V. N. Vasilets, A. V. Kuznetsov, and V. I. Sevastianov, J. Biomed. Mater. Res., 69A, 428 (2004). 
10. K. Okudaira, S. Hasegawa, P. T. Sprunger, E. Morikawa, V. Saile, K. Seki, Y. Harada, and N. Ueno, J. Appl. Phys., 83, 4292 (1998).

11. A. Gupta, R. Liang, F. D. Tsay, and J. Moacanin, Macromolecules, 13, 1696 (1980).
12. H. Hu and D. Chen, Eur. Polym. J., 30, 1243 (1994).

13. A. Hozumi, T. Masuda, K. Hayashi, H. Sugimura, O. Takai, and T. Kameyama, Langmuir, 18, 9022 (2002).

14. S. Tanaka, Y. Naganuma, C. Kato, and K. Horie, J. Photopolym. Sci. Technol., 16, 165 (2003). 